\title{
Review of the Location Technology in Underground Mines
}

\author{
Pei Liang ${ }^{1,2}$ a , Liu Shixuan1, 2,b , Wang Bo ${ }^{1,2, c}$, Li Wenqing ${ }^{1,2, d}$ \\ ${ }^{1}$ Shandong Provincial Key Lab of Ocean Environment Monitoring Technology, Qingdao, China \\ ${ }^{2}$ Institute of Oceanographic Instrumentation, Shandong Academy of Science, Qingdao, China \\ apeiliang2002@163.com, ${ }^{b} 321$ go@sohu.com, ${ }^{\mathrm{c}}$ bob80.wang@hotmail.com, ${ }^{\mathrm{d}}$ livenson@163.com
}

Keywords: coal mine, mine tunnel, location system, WLAN

Abstract: The development of the coal mine location systems in the recent decades was reviewed. Location technologies about IR, RFID, zigbee, leakage communication, through-the earth communication and WLAN in mine tunnels were mainly discussed. The conclusion that technologies based on WLAN or zigbee may be the better choices for underground mines.

\section{Introduction}

China accounts $35 \%$ of the world coal production, but the death toll accounts almost $80 \%$ of the world, and the situation of the coal mine safety production is serious. Actually, the rescue time are often delayed because the location of the miners cannot be judged in time, and this is one of the major factors of the high death rate in China.

So, accurate and real-time location systems are important to the coal mine industry. This paper is a review about the coal mine location systems in the recent decades.

\section{Present State}

Location technologies based on the mobile network and GPS [1] are the major schemes to the location problem. But mine tunnels are deeply under the ground, signals from the mobile network and GPS or A-GPS cannot be received, so cannot be used in coal mine tunnels. Different technologies should be adopted to solve the location problem in mine tunnels.

The technologies can be used in underground tunnels includes IR, ultrasonic ranging, Bluetooth, RFID, zigbee, UWB, leakage communication, through-the earth communication and WLAN, and in these technologies, location was realized by some special algorithms based on the received signals from the network. We will mainly talk about some of them.

IR. In IR technologies, the mobile objects transmit the IR signals modulated with the mobile ID, and geolocation was realized by the IR sensor array installed indoors. And the use of IR in mines can be traced back to 1991, the Finsch mine in south Africa used a underground mine scraper automatic location system [2]. In this system, a token ring network composed by IR sensors was adopted, and the IR sensors were installed in the important locations of the mine. When scrapers passed such IR sensors, the IR transmitter installed on it will transmit an IR signal modulated with the ID of the scraper, and this signal will be received by the IR sensor array, then be uploaded to the monitoring center, and be located finally.

The Active Badges system [3] developed by AT\&T is a indoor geolocation system based on IR, and it also can be used in mine tunnels. This system was composed by badges and sensor array installed in the places previously decided, everyone wears a badge which transmits an certain ID signal, the sensor array will receive this IR signal and then transmit to the monitoring computer, and the monitoring computer will decide the object's location. 


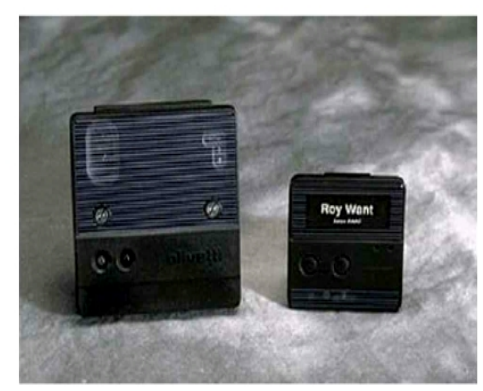

Fig. 1 the base station and badge in Active Badge

The drawbacks of the IR technology can be concluded as: (1) the effective distance is limited, so a high-density of receiving sensor array is required and the system cost and the maintenance cost is expensive; (2) IR signals have strong direction, the signal can be received only at the propagating direction; (3) only coarse location is realized, that is to locate the object in a certain space range.

RFID. In RFID(Radio Frequency Identification) technology, the ID of the object is modulated on a RF wave, when the object is within the covering range of the antenna of the receiver, the object can be detected and located. In 1994, the Premier mine installed such a system[4], in which a monitor is installed on the object, and the monitor is always detecting the RF tags installed in the important places, and the tags transmit RF signals modulated with the certain ID, here the transmit distance is more than $3 \mathrm{~m}$. And this is the early application of RFID in mines. In 1995, another mine in Australia developed a miner detect system based on RFID to check if there were miners in the dangerous places [5]. In this system, antennas installed on the tunnel roof were used to detect the small tags in the miners' helmet, so the situation of the miners in the range covered by the antennas can be determined. In recent years, China also developed such systems.

Location systems based RFID can be used in severe environment, but RFID technology can only locate objects in a range scale, that is, to locate objects in the range between two receivers, and if we want to achieve more precise location, high density base station network is required, and the system complexity and cost will be high.

Leakage Communication. Leakage communication uses radiation guide leakage coaxial cable instead of normal antennas in base station, and leakage coaxial cable can be seen as distributed antennas, as shown in Fig.2. The mobile near the cable communicate to the base station by the coupling to the cable, and communication between two mobile stations is also depend on the leakage cable. And because the transmission loss is high, the communication distance between the mobile station and base station is no longer than $2500 \mathrm{~m}$, and usually $500 \mathrm{~m}$, so two-way repeater is often used to extend the communication distance.

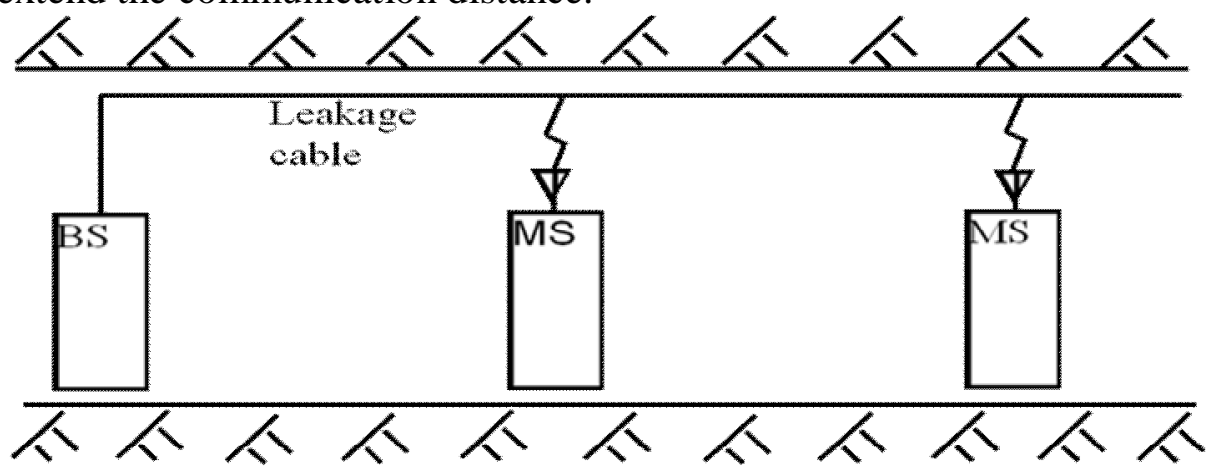

Fig.2 leakage communication

Now, sophisticated leakage systems mainly including Flexcom and MultiCOM of Canada, TR-150VHF and TR-500UHF of Tunnel Radio of America Inc. And Flexcom includes a subsystem: FlexTag, FlexTag know who are in underground tunnels, and at which mine area does he work. Also MultiCOM developed miner tracking module Plexpass, which can monitor the pass and safe condition of the workers. 
To Leakage communication, the tunnel shape, cross section, roughness, branches, bending inclination, rock structure, medium and tunnel support have small influence, so the communication channel is stable and the magnetic influence is small.

However, leakage communication is not reliable and robust. In leakage communication the communication between two mobile station have to be transformed by the base station, so if the base station breakdown, the whole system will breakdown, and any relay and cable fault will cause the system after the relay breakdown. And in mine tunnel, the environment is critical, the failure rate of the relay and cable is high, so in mines covered several kilometers, equipment maintenance will be very difficult. Therefore, leakage communication is not a perfect technology for location.

ULF/VLF through-the-earth communication. ULF/VLF through-the-earth communication utilizes the earth as the propagation media. In 1990, an Australian mine developed the PED(Personal Emergency Device) system based on ULF/VLF through-the-earth communication. The signal firstly is amplified by high power transmitter, and then be transmitted by the ring antenna, tens of kilometers long, and the signal will propagate through the earth to the receivers under mines, as shown in Fig.3. Lots of mines in China have installed PED.

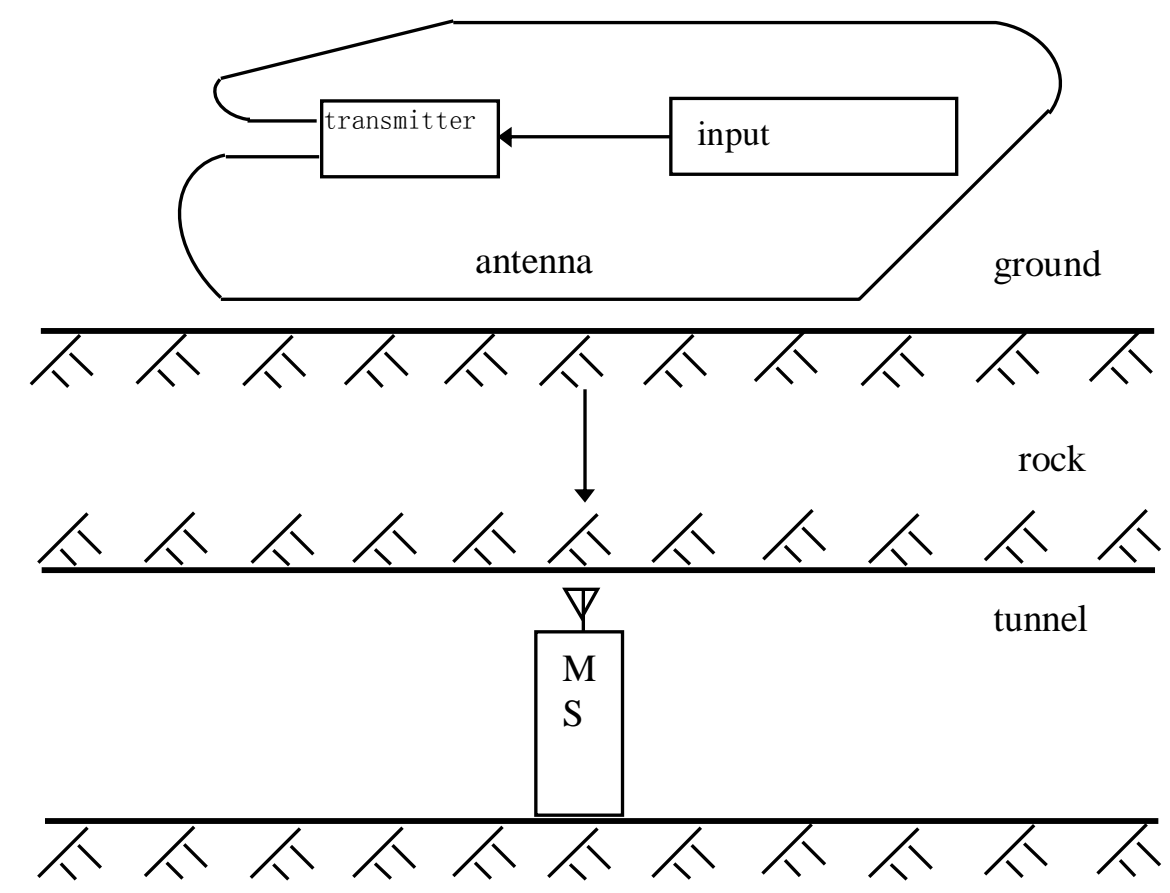

Fig.3 through-the-earth communication

In this technology, the signal input, transmitter, transmit antenna are on the ground, so when accidents happened underground, the system can still work. And the drawbacks of VLF/ULF through-the-earth communication is the following:

(1) One way communication: the size of the transmit antenna is very large and its transmit power is up to several kilo-watt, so it's difficult to develop intrinsic safe antenna. Therefore, this technology only can be used to one way communication, that is, the antenna on the ground transmits signals and the receivers under mines always receive signals.

(2) High Magnetic influence: the space in mine tunnels is small and limited, and the electrical machines are relatively concentrated, and this result to serious magnetic influence in tunnels, especially that the $50 \mathrm{~Hz}$ interference influence the VLF/ULF through-the-earth communication very much.

(3) Critical construction.

So, through-the earth communication is often used in dispatching and accident auxiliary communication system, and its one way communication method make the monitor on the ground incapable to know the location of the miners underground. 
WLAN. With the development of science and technology, WLAN is more and more popular $[6,7,8]$. Location technology based on WLAN is attaching more and more attention.

WLAN-based location system firstly was only used in indoor location, lots of such systems were developed such as RADAR(2000) of Microsoft Research [7], Aura(2001) of Carnegie Mellon university, Wi-Fi Watch-dog of Newbury Networks in Boston, location system based on IEEE802.11 and HIPERLAN proposed by Ekahau, and so on.

With the development of the Wi-Fi network and Voice over IP, WLAN-based location technology was applied in mine tunnels [9-14]. In 2006, Ekahau of USA developed Wi-Fi location tags T201 for miner locating in mine tunnels. Now T201 has been used in different mines of Spain, South America, Africa and Europe. And both Ekahau and RADAR(2000) were finger-printing technologies. Drawbacks of such technology was obviously: (1) Prior work is complex, each measuring point should be measured and then a large database should be developed, and the location accuracy was decided by the density of the measuring points. So for complex and large range conditions such as mine tunnels, finger-printing is not applicable. (2) only applicable for static conditions, because the data measured is static, and the environment is dynamic, even in the same location point, the data measured in real time will be very different to the data in the database, and this will result to great location error.

L3 Technology, Inc. in Canada developed a wireless mine management and safty system to realize the location and management of the miners underground, Wi-MMS ${ }^{\mathrm{TM}}$. In Wi-MMS ${ }^{\mathrm{TM}}$, commercial and sophisticated RF router was adopted, the wireless network can be setup easily.

AeroScout and NL Technologies proposed Wi-Fi 802.11-based RFID tag T2, and then furtherly developed T2-based application for mines: RTLS(real time location solution). RTLS is a different technology, and it adopts the real-time RSSI and TDOA to realize location, so RTLS can track the dynamic environment and higher location accuracy can be achieved. Now, RTLS has achieved a $10 \mathrm{~m}$ location accuracy. However, in the serious environment of indoor or mine tunnels, to realize precise location using RSSI and TDOA is still difficult.

Besides the technologies discussed above, there are some other wireless technologies that have been applied in under-ground mines, but in the end not applicable for their drawbacks, for example, wire inductive communication, and intermediate frequency wireless communication. For wire inductive communication, a single dedicated wire should be installed along the tunnel, and communication was realized by magnetic induction. The drawback of wire inductive communication is that the factors such as earth medium will make the parameters unstable, and is susceptible to interference at low frequency, so will lead to a drop in the quality of communication. Intermediate frequency wireless communication utilize the existing metal medium in tunnels to transmit intermediate frequency signals, and its main drawback is that the communication distance is limited, and the communication distance and quality is not stable for the different metal medium condition in tunnels.

\section{Conclusion}

To the under-ground mines, WLAN may be a better choice, and in the standard TCP/IP system the different telemeters and sensor network can be easily integrated into the WLAN. Moreover, the distribution of mine tunnels is very complicated, there are lots of bending branches, and the bending branches are often in different levels, which will greatly attenuated the signal, so the WLAN-based micro-cell network is applicable in underground mines. And in such a standard network, all the different network underground can be integrated, so the cost of the installation, maintenance and management can be reduced, and the efficiency can be increased.

\section{Acknowledgements}

This work was financially supported by special funds for scientific research projects of Marine public welfare industry: (201405022-2). 


\section{References}

[1] E. D. Kaplan. Understanding GPS: Principles and Applications, Artech House, 1996.

[2] R. A. LUKE. Diamonds with dispatch: computerized LHD dispatching at De Beers Finsch-A progress report. World mining Equipment, 1992(6):24 28.

[3] R. Want et al. The Active Badge Location System, ACM Trans. Information Systems, 1992(1): 91 102.

[4] Anon. Safety in microprocessors-TIRIS tracking, World mining Equipment, 1995(5):44.

[5] D. J. Hind. Application of radio frequency identification systems in the mining industry. $6^{\text {th }}$ Canadian Symposium on Mining Automation, Montreal, 1994:34 39.

[6] Arvinder Singh. LOCUS: Wireless LAN Location Sensing, PhD dissertation, Worcester Polytechnic Institute, 2004.

[7] P. Bahl, V. Padmanabhan. RADAR: An In-Building RF Based User Location and Tracking System, IEEE INFOCOM, Israel, 2000(2):775 784.

[8] Garlan D., Siewiorek D.P., Smailagic A. et al. Project Aura: toward distraction- free pervasive computing. Pervasive Computing, IEEE, 2002, 1(2):22 31.

[9] D. C. Reid, D. W. Hainsworth, J. C. Ralston, et al. Shearer Guidance: a Path towards Longwall Automation, Proceedings of the 1st International Workshop on Robotics for Mining and Underground Applications: Brisbane, Australia, 2000.

[10] M. S. Kelly, P. Lever, D. W. Hainsworth, et al. Longwall Automation: Are We Ready for Success? Proceedings of the 3rd International Under-ground Coal Conference, Manly, Australia, 2001:344-355.

[11] Garry Einicke1.The Application of Wireless LANS in Mine Automation, Leveraging wireless technology within existing business models for corporate advantage Sydney, 2002:1 10.

[12] Hargrave C.O., Ralston J.C., Hainsworth, D.W. Optimising wireless LAN for longwall coal mine automation, Industry Applications Conference, 2005. Fourtieth IAS Annual Meeting, 2005(1):218 224.

[13] Huber, J.F., Mobile next-generation networks, IEEE Trans. Multimedia, 2004, 11(1):72 83.

[14] Yi Qian Hu, Hsiao-Hwa Chen; A call admission control framework for voice over WLANs, IEEE Trans. on Wireless Communications, 2006, 13(1):44 50. 